\title{
Flexibler Gebäudebetrieb durch speichergestützte Lastverschiebungen
}

\author{
G. Zucker OVE
}

Online publiziert am 30. November 2013

(C) Springer Verlag Wien 2013

Ein Drittel des weltweiten Energieverbrauchs und der zugehörigen Emissionen lässt sich auf den Gebäudesektor zurückführen. Trotz dieser prominenten Position spielen Gebäude in modernen Energienetzen eine eher passive Rolle. Während Industrie und Transport als aktive Partner in das Energiesystem eingebettet werden, sind Gebäude unidirektionale Endpunkte ohne Steuermöglichkeiten. Aktive Teilnehmer eines Smart Grid hingegen können zur Optimierung des Gesamtsystems beitragen, indem sie flexibel betrieben werden und Informationen mit dem Netz austauschen.

Gebäude beherbergen eine Reihe signifikanter Energie verbrauchender Prozesse, vor allem im Bereich Heizung, Lüftung, Klima und Kühlung. Viele der Prozesse haben operative Bandbreiten bei Sollwerten und Einsatzzeiten, die bei Bedarf ausgenützt werden können. Diese Flexibilitäten erlauben eine Optimierung des Gebäudebetriebs in verschiedene Richtungen.

Bis heute ist lastseitige Automatisierung fast immer gleichzusetzen mit Lastabwurf: Vertraglich geregelt werden Verbraucher, deren Prozesse temporäre Unterbrechungen erlauben (z. B. Pumpen oder Belüftungsanlagen), über ein ausgestrahltes Signal abgeschaltet. Je nach Modell dauert diese Unterbrechung eine definierte Zeitspanne oder wird mit weiteren Signalen aufgehoben. Hier kann mit IKTMethoden angesetzt werden, indem mehr Intelligenz in die Optimierung der Gebäudeprozesse eingebracht wird. Durch eine Kombination aus Gebäudeautomation und Kommunikation mit dem elektrischen Netz können Lastverschiebepotenziale gehoben werden. Dazu müssen flexible, generische Lastmodelle für Gebäude entwickelt und in eine interoperable Kommunikationsinfrastruktur eingebettet werden. Den Grundstein für die Unterstützung des elektrischen Netzes bilden die Lastverschiebungen, die durch Ändern von Schaltzeitpunkten, Dauer oder Sollwerten von elektrischen Lasten im Gebäude aktiviert werden. Das energetisch größte Potenzial im Gebäude haben thermische Lasten wie Heizung, Lüftung und Kühlung, die durch Veränderung von Raumtemperaturen oder Sollwerten in den thermischen Anlagen beeinflusst werden können (andere Stromverbraucher wie Beleuchtung oder Lasten im direkten Einflussbereich der Bewohner - z. B. Waschmaschinen oder Kühlschränke - werden nicht untersucht). Dabei darf der Komfortbereich für die Bewohner durch die Optimierung nicht verlassen werden, was eine Möglichkeit zur Vorhersage des thermischen Gebäudeverhaltens erfordert. Dies wird durch thermische Simulation des Gebäudes erreicht, indem die relevanten Zeitkonstanten für Erwärmung und Abkühlung des Gebäudes identifiziert werden. Damit wird eine Vorhersage erstellt, wie lange z. B. die Heizung abgeworfen werden kann.

Die zusätzliche Gebäudeintelligenz wird in Form eines Building Agent zur Verfügung gestellt. Diese Software bildet als Teil des Gebäudeautomatisierungssystems die Schnittstelle zum Kommunikationspartner im elektrischen Netz. Der Kern des Building Agent ist die Flexibilitätslogik zur Ermittlung der Lastverschiebepotenziale. Diese Logik berechnet bei einer Anfrage durch das zentrale Netzmanagement auf Basis der Klimaprognose sowie eines spezifischen Gebäudemodells die Möglichkeiten zur Lastverschiebung. Im ersten Schritt wird die geplante Lastverschiebung dazu in die Phasen Normalbetrieb, Preconditioning, Peaktime und Rebound eingeteilt. Basis für die Berechnung des Verschiebepotentials bildet der Normalbetrieb des Systems, also der Betrieb ohne den Einfluss durch den Building Agent bzw. des übergeordneten Netzmanagements. Im Vorfeld einer (geplanten) Lastverschiebung kann eine „Vorbereitung" des Systems erforderlich sein, die als Preconditioning-Phase bezeichnet wird. Nach der Phase der eigentlichen Verschiebung (Peaktime) kann bis zur Rückkehr in den Normalbetrieb noch die so genannte Rebound-Phase auftreten.

Wird eine durch die Flexibilitätslogik berechnete Verschiebung durch das zentrale Netzmanagement bestätigt, wird ein entsprechender Zeitplan zur Steuerung der Anlage erstellt und an die Steuerlogik weiter gegeben. Diese gibt zu Beginn der jeweiligen Phasen Befehle an das Gebäudeautomationssystem weiter.

Das daraus entstehende Flexibilitätspotenzial kann als Netzdienstleistung zur Unterstützung des elektrischen Netzes angeboten werden. Durch die Möglichkeit, den Verbrauch an die Erzeugung anzupassen, wird außerdem die Möglichkeit geboten, mehr erneuerbare Energieerzeuger in den Netzbetrieb zu integrieren (Abb. 1).

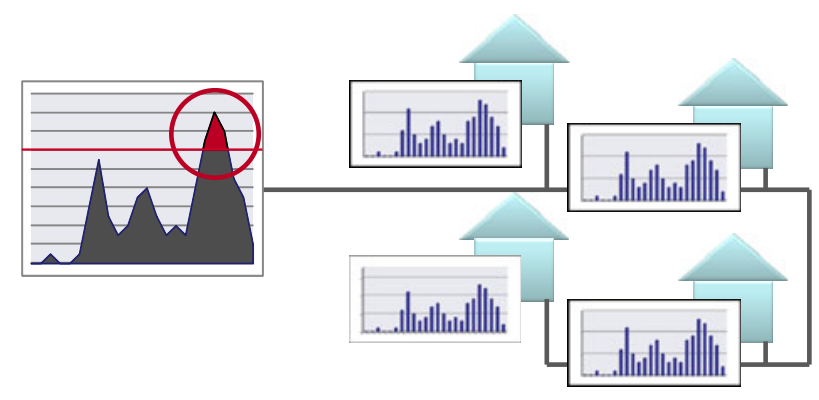

Abb. 1. Lastspitzen im elektrischen Netz

Kurzfassung eines Vortrags bei der 51. Fachtagung der Österreichischen Gesellschaft für Energietechnik im OVE, die am 10. und 11. Oktober 2013 in Graz stattfand.

Zucker, Gerhard, Energy Department, Sustainable Building Technologies, AIT Austrian Institute of Technology, Giefinggasse 2, 1210 Wien, Österreich

(E-Mail: gerhard.zucker@ait.ac.at) 